Nedad Memić*

Wien

\title{
BESONDERHEITEN DER DEUTSCHEN VERWALTUNGSSPRACHE IM K. U. K. BOSNIEN-HERZEGOWINA
}

\section{EINFÜHRUNG}

Die österreichisch-ungarische Okkupation Bosnien-Herzegowinas im Jahre 1878 änderte den Charakter der bosnisch-herzegowinischen Verwaltung tiefgehend. Während Österreich-Ungarn relativ wenig tat, um die aus der osmanischen Zeit geerbte wirtschaftlich-soziale Struktur Bosnien-Herzegowinas grundlegend zu ändern - besonders was den Anteil der Landwirtschaft an der Wirtschaftsstruktur des Landes und den Status der Leibeigenen betraf -, konzentrierte sich die neue Okkupationsmacht auf andere Bereiche, vor allem

[...] auf die völkerrechtliche Einbindung der neuerworbenen Länder in das dualistische Staatsgefüge, auf die zügige Einführung einer modernen Verwaltung sowie auf die technische Modernisierung, vor allem durch den Ausbau des Verkehrsnetzes und der Industrie und schließlich auf die Schaffung einer der Dynastie treuen Elite durch die Eingriffe in den Konfessions- und Kulturbereichen. (Džaja 1994: 42)

Einige der größten Verwaltungsvorhaben der österreichisch-ungarischen Zeit waren die Eingliederung Bosnien-Herzegowinas in das gemeinsame Zollgebiet der Donaumonarchie im Jahre 1880 und die Einführung der allgemeinen Wehrpflicht im Jahre 1881. Damit war Bosnien-Herzegowina - de facto ein Okkupationsgebiet, das völkerrechtlich bis zur Annexion 1908 Teil des Osmanischen Reiches war - in den Verwaltungsapparat Österreich-Ungarns eingeschlossen.

Während die österreichisch-ungarische Verwaltung die administrative Aufteilung Bosnien-Herzegowinas in Kreise (zur osmanischen Zeit Sandschak) und Bezirke (osmanisch Kaza) aus der osmanischen Zeit weitgehend behielt, hatte sie wenig Vertrauen in die Beamtenschaft aus der osmanischen Zeit. Aus diesem Grund erfolgte die Besetzung der Beamtenposten fast ausschließlich aus den Reihen der Zuzügler aus beiden Teilen der Donaumonarchie, die Zahl der Beamten stieg bis 1908 auf mehr als 9.500 Personen, in der osmanischen Periode gab es vergleichsweise nur 120 Beamte (vgl. Malcolm 2011: 255).

\section{ZUR SPRACHFRAGE IM K. U. K. BOSNIEN-HERZEGOWINA}

Die ersten Standardisierungsversuche des einheimischen Idioms in Bosnien-Herzegowina fallen in die 1860er Jahre des 19. Jahrhunderts. Der liberale osmanische Statthalter

nedad.memic@gmail.com 
(valija) in Bosnien-Herzegowina Topal Osman-Pascha ließ neue muslimische und christliche Schulen im Lande errichten, verbesserte die Infrastruktur, eröffnete die erste Bahnlinie von Banja Luka bis zur kroatischen Grenze und das erste öffentliche Spital (heute immer noch unter dem Namen hastahana bekannt) in Sarajevo (Malcolm 2011: 239). Unter Topal Osman-Pascha wurde aber auch die erste moderne Druckerei in Bosnien-Herzegowina im Jahre 1865 errichtet, die 1866 in den Besitz der Provinz (vilajet) überging und in der die ersten bosnisch-herzegowinische Zeitungen „Bosanski vjestnik“ („Der Bosnische Bote“) und „Bosna“ gedruckt wurden (Neweklowsky 2004: 29).

Im „Bosanski vjestnik“ wurde die reformierte kyrillische Schrift des serbischen Sprachreformators Vuk Stefanović Karadžić (inkonsequent) angewendet - damit war Bosnien-Herzegowina das erste südslawische Land, das die reformierte phonologische Schrift Karadžićs inoffiziell einführte (Okuka 1998: 52). Die Sprache wurde jedoch offiziell „Bosnisch“ genannt, was zur osmanischen Zeit die amtssprachliche Bezeichnung für die Landessprache in Bosnien-Herzegowina war (Okuka 1998: 47). Parallel zur Bezeichnung „Bosnisch“ fanden bei Serben und Kroaten die nationalen Sprachbezeichnungen „Serbisch“ und „Kroatisch“ immer mehr Anwendung. Während in den ersten Jahren der österreichisch-ungarischen Okkupation eine programmatische Unsicherheit herrschte, wie das einheimische Idiom in Bosnien-Herzegowina zu benennen wäre - es kursierten die Bezeichnungen „Kroatisch“, „Serbisch“, „bosnische Landessprache" - bürgert sich seit den frühen 1880er Jahren unter der Politik des Gemeinsamen österreichisch-ungarischen Finanzministers und Verwalters von Bosnien-Herzegowina Benjamin Kállay die ofizielle Sprachbezeichung „Bosnisch“ ein. Obwohl die Bezeichnung „Bosnisch“ hätte bedeuten können, dass die Sprachpolitik im österreichisch-ungarischen Bosnien-Herzegowina darauf abzielte, die dortige Sprache als eine vom Serbischen und Kroatischen unabhängigen Standardvarietät zu etablieren, war das im Großen und Ganzen nicht der Fall. 1883 wurde als Resultat der Aktivitäten einer von der Landesregierung ernannten Sprachkommission die phonologische Rechtschreibung Vuk Karadžićs in Bosnien-Herzegowina offiziell eingeführt (Okuka 1998: 52), Bosnien-Herzegowina akzeptierte zudem die neustokawische ijekawische Dialektvarietät - damals auch ,južno narječje“ (der südliche Dialekt) genannt - als standardsprachliche Basis (Šator 2004: 84). Auf diesen Postulaten wurde auch die Grammatik der bosnischen Sprache aus dem Jahr 1890 veröffentlicht. Die österreichisch-ungarische Zeit in Bosnien-Herzegowina war aus diesem Grund eine wichtige Epoche in der Standardisierung des einheimischen Idioms, obwohl Österreich-Ungarn die Sprachfrage in Bosnien-Herzegowina als Teil seiner politischen Strategie auf dem Balkan betrachtete. Diese Strategie war in erster Linie darauf ausgerichtet, das dualistische System der Monarchie zu stärken, indem man einerseits versuchte, serbische und kroatische nationale Bestrebungen einzudämmen, und sich andererseits zum Teil auf die bosniakisch-muslimischen Eliten stützte, die sich wiederum gegen den aufkeimenden serbischen und kroatischen Nationalismus stemmten.

Ab 1907 wurde die offizielle Bezeichnung der Landessprache von „Bosnisch“ auf „Serbo-Kroatisch“ geändert - was zugleich das Scheitern der von Kállay propagierten integralen Bosniertums bedeutete. Bosnien-Herzegowina war damit das erste 
südslawische Land, dessen Sprache offiziell „Serbo-Kroatisch“ hieß. Sprachlich änderte sich jedoch wenig: Im öffentlichen Gebrauch wurden sowohl kyrillische als auch lateinische Schrift verwendet, während nach dem Ausbruch des Ersten Weltkrieges die kyrillische Schrift 1915 aus politischen Gründen offiziell verboten wurde (Šipka 2001: 29).

\section{DEUTSCH ALS AMTSSPRACHE IM K. U. K. BOSNIEN-HERZEGOWINA}

Die Okkupation Bosnien-Herzegowinas im Jahre 1878 wirkte sich aber nicht nur auf die Landessprache aus. Insbesondere mit dem Zuzug zahlreicher Monarchiebürger nach Bosnien-Herzegowina und der Eingliederung des Landes in das Zollgebiet und in das Militärsystem der Monarchie gewannen andere Sprachen, insbesondere das Deutsche, an Bedeutung.

\subsection{Deutsch im Verwaltungsapparat}

Auf die Stellung des Deutschen im k. u. k. Bosnien-Herzegowina wirkte sich in erster Linie der spezifische Status des Landes im staatlichen Gefüge Österreich-Ungarns aus. Von 1878 bis 1908 war Bosnien-Herzegowina völkerrechtlich ein Okkupationsgebiet. Die Zivilverwaltung auf der Landesebene hatte in der gesamten Okkupations- und später in der Annexionszeit den militärischen Charakter - der Landeschef in der Landesregierung Bosnien-Herzegowinas wurde nämlich immer aus dem Militär ernannt (Džaja 1994: 42). Im Unterschied zu den anderen Kronländern war Bosnien-Herzegowina als Okkupationsgebiet keinem der beiden Reichshälften unterstellt, sondern direkt aus Wien verwaltet: Von 1879 bis zum Ende der österreichisch-ungarischen Zeit war das Gemeinsame Finanzministerium für die Angelegenheiten Bosnien-Herzegowinas zuständig: zuerst als Okkupationsgebiet, dann ab 1908 als Reichsland.

Während der Okkupationszeit gab es in Bosnien-Herzegowina keine einheitlichen Regelungen über den Amtssprachengebrauch (Juzbašić 2002: 390). Da Österreich-Ungarn eine multinationale und mehrsprachige Monarchie war, kam diese Mehrsprachigkeit auch in Bosnien-Herzegowina zur Geltung. Historiker unterscheiden hier zwischen dem inneren und äußeren Gebrauch der Amtssprache. Unter dem inneren Amtssprachengebrauch versteht man die Sprachverwendung innerhalb einer Behörde bzw. zwischen den Behörden oder unterschiedlichen Verwaltungsinstanzen. Der äußere Amtssprachengebrauch umfasste die Kommunikation der Behörden mit den Parteien. In Bezug auf die so genannte äußere Amtssprache wurde generell vorgeschrieben, dass man sich in der Kommunikation mit den Parteien der Landessprache bedient, sofern die Parteien diese auch verstehen. Die Parteien konnten wiederum ihre Anträge an die Behörden nicht nur in bosnischer, sondern auch in deutscher, ungarischer und sogar türkischer Sprache stellen. Ähnliches konnte man auch im Justizwesen beobachten: Mit den Parteien hatte man in der Landessprache zu korrespondieren, jedoch konnten die der Landessprache nicht mächtigen Bürgerinnen und Bürger auch Deutsch oder Ungarisch verwenden (Juzbašić 2002: 390f.).

Während man im äußeren Amtssprachegebrauch Bosnisch forcierte, etablierte sich Deutsch im inneren Amtssprachengebrauch. So wurde ab 1879 geregelt, dass die 
Korrespondenz der Landesregierung mit allen Verwaltungsebenen, die unterhalb der Kreise stehen, in der Landessprache zu erfolgen ist. Mit allen höheren Verwaltungsebenen musste die Korrespondenz auf Deutsch geführt werden. Ab 1881 war Deutsch auch die alleinige innere Amtssprache des Justizwesens, alle Gerichtsbediensteten mussten demnach der deutschen Sprache mächtig sein. (Juzbašić 2002: 392f.). Deutsch war auch die Sprache, die die Zivilverwaltung in der Korrespondenz mit dem Militär verwendete. Dazu war Deutsch die Sprache, in der die bosnisch-herzegowinische Landesregierung mit allen Ministerien, Militärkommandos sowie anderen Landesregierungen der Monarchie zu korrespondieren hatte, ab 1897 musste die Korrespondenz mit den ungarischen Ministerien auf Ungarisch erfolgen (Juzbašić 2002: 393f.).

Obwohl der Amtssprachengebrauch relativ flexibel war und sich an den tatsächlichen Bedürfnissen orientierte, kann man zusammenfassend feststellen, dass Deutsch seine Rolle als innere Amtssprache bzw. Amtssprache der höheren Verwaltungsebenen einnahm, während die Landessprache die äußere Amtssprache bzw. die Amtssprache der niedrigen Verwaltungsebenen war. Wurde die jährliche Sammlung der Gesetze und Verordnungen für Bosnien und die Herzegowina von 1878 bis 1880 ausschließlich auf Deutsch publiziert, wurde sie ab 1881 zweisprachig - deutsch und bosnisch - herausgegeben.

\subsection{Deutsch in Schulwesen und Presse}

Die Bedeutung des Deutschen in der damaligen Zeit kann man auch an seiner Position als Fremdsprache beurteilen. So war Deutsch für die meiste Zeit der österreichisch-ungarischen Verwaltung in Bosnien-Herzegowina die erste und oft die einzige Fremdsprache, die man im Schulwesen unterrichtet hat: Dies galt jedenfalls für die staatlichen Schulen (Radanović 2013: 114). In den neugegründeten Gymnasien bzw. Realgymnasien hat man die Zahl der Wochenstunden in Deutsch allmählich vergrößert. So betrug ab 1910 bis Ende der k. u. k. Zeit die Zahl der Wochenstunden in den großen Realgymnasien in Sarajevo und Banja Luka in der ersten und zweiten Klasse jeweils fünf Wochenstunden, in der dritten Klasse vier Wochenstunden und von der vierten bis zur achten Klasse jeweils drei Wochenstunden in Deutsch (Radanović 2013: 119f.). In den Handelsschulen und in der 1912 eröffneten Handelsakademie in Sarajevo war Deutsch sogar das Fach mit den meisten Wochenstunden (Radanović 2013: 121).

Deutsch war auch die Sprache der Presse im k. u. k. Bosnien-Herzegowina. So verzeichnet Džaja unter offiziellen und nationalpolitisch neutralen Blättern 22 Zeitungen, die in deutscher Sprachen bzw. zwei- oder mehrsprachig (meistens deutsch und bosnisch bzw. serbokroatisch) publiziert wurden (Džaja 1994: 93f.). Das waren unter anderem amtliche und halbamtliche Blätter, Blätter für Politik und Wirtschaft sowie wissenschaftliche und berufsständische Blätter. Die Überlebensdauer dieser Blätter war unterschiedlich, die wohl bekannteste und langlebigste deutschsprachige Zeitung der k. u. k. Periode in Bosnien-Herzegowina war die „Bosnische Post“". Die Zeitung ist von 1884 bis 1918 erschienen, meistens drei bis vier Mal in der Woche, ab 1896 wurde sie täglich publiziert. Die Blattlinie der „Bosnischen Post“ galt als regierungsnah. 


\section{DAS GEMEINDESTATUT VON SARAJEVO (1884)}

Da sowohl der völkerrechtliche Status Bosnien-Herzegowinas als auch der dortige Verwaltungsapparat der österreichisch-ungarischen Monarchie eine Reihe von Besonderheiten im Vergleich zu den anderen Kronländern der Donaumonarchie bzw. zu den restlichen deutschsprachigen Ländern aufwies, ist es auch zu erwarten, dass die deutsche Amtssprache in Bosnien-Herzegowina diese Besonderheiten widerspiegelte. Sie ergaben sich vor allem aus dem Sprach- und Kulturkontakt mit dem Bosnischen und änderten sich auch in unterschiedlichen Phasen der österreichisch-ungarischen Präsenz in Bosnien-Herzegowina.

Als Primärquelle für die Literatur ziehen wir das im Jänner 1884 offiziell veröffentlichte Gemeindestatut für die Stadt Sarajevo heran. Dieses wurde zweisprachig deutsch und bosnisch - in der Sammlung der Gesetze und Verordnungen für Bosnien und die Herzegowina (Jahrgang 1884) verfasst. Während die österreichisch-ungarische Verwaltung die osmanischen Kreise und Bezirke weitgehend beibehielt, war eine Neuorganisation von Gemeinden notwendig. So war nun die Landeshauptstadt Sarajevo als eine Gemeinde organisiert, die wiederum in einzelne Bezirke eingeteilt war. Eine gewisse Ähnlichkeit mit der Verwaltungsstruktur von Wien und anderen Monarchiestädten ist also feststellbar.

Die Analyse der Sprache im Gemeindestatut bezieht sich auf mehrere sprachliche Ebenen: In diesem Zusammenhang konzentrieren wir uns konkret auf orthografische, morphologische, lexikalische und sprachkontaktbezogene Besonderheiten.

\subsection{Orthografische und morphologische Besonderheiten}

Die Orthografie des Gesetzestextes entspricht dem deutschen Orthografieusus in der zweiten Hälfte des 19. Jahrhunderts vor der Orthografie-Reform von Duden. Dies bezieht sich insbesondere auf die $c$-Schreibung bei Lexemen fremder (meist lateinischer) Herkunft wie Advocat, Cassa, Commissär, Concurs, Confession, local usw., die th-Schreibung in Lexemen wie Berathung, Gemeinderath, Thätigkeit, theilnehmen, Werth usw. oder die ss-Schreibung bei ausserordentlich, Gemässheit, Mass, unregelmässig; Geheimniss, Gelöbniss usw.

Dazu ist eine nicht konsequente Schreibung des verbalen Suffixes -ieren bzw. der deverbalen Ableitungen mit diesem Suffix zu beobachten: Approvisionirung, scontriren, aber Bequartierung, Landesregierung usw.

Im morphologischen Bereich ist eine konsequente Verwendung des Suffixes - $e$ im Dativ Singular nach dem ostmitteldeutschen Modell zu beobachten: in dem Gemeinde-Statute, dem Gemeinderathe, im letzten Quartale usw.

\subsection{Lexikalische Besonderheiten}

In der Neuorganisation der Gemeindeverwaltung in Bosnien-Herzegowina (hier konkret in Sarajevo) griff man zur österreichisch-ungarischen Verwaltungsorganisation und somit auch zu ihrer Terminologie zurück. Dazu finden wir im Korpus u. a. folgende Beispiele: Agenda, Gassenbezeichnung, Gebahrung, Gemeinderath, 
Gemeinderathswahlen, Jahresbudget, Landesregierung, Landessprache, Magistratsbeamter, Sanitätswesen, Stadtbezirk, Stadtmagistrat usw.

Im Gesetzestext existiert auch eine Reihe von Lexemen aus dem österreichischen Verwaltungswortschatz, die heute als veraltet, unüblich oder ungebräuchlich gelten, u. a.: Advocat, Arrest, Approvisionirung, Approvisionirungswesen, Bequartierung, Civil-Adlatus, Concurs, Conscription, Controlsrecht, Corporation, Ehrenbürgerrecht, Executivorgan, Gemeindecasse, Heimatsrecht, Inslebentreten, Majorität, Regierungs-Commissär, Remuneration, Revennue, scontriren, Scontrirung, Strafsanction usw.

\subsection{Kontaktsprachliche Phänomene}

Die deutsche Verwaltungssprache in Bosnien-Herzegowina übernahm auch einige landes- bzw. kulturspezifische Lehnwörter aus dem Bosnischen. Im Text des Gemeindestatuts sind zwei solche Lehnwörter vorhanden: Bezirks-Muktar und Mahala. Beide sind ins orthografische und grammatische System des Deutschen integriert und werden ohne zusätzliche Erklärung oder Übersetzung verwendet: So existieren im Text auch flektierte Formen wie Mahalas (Nominativ Plural) oder des Bezirks-Muktars (Genitiv Singular) usw. Beide Lexeme sind eigentlich Orientalismen im Bosnischen und waren bereits in der osmanischen Zeit halboffizielle bzw. offizielle administrative Termini. So war Sarajevo zur osmanischen Zeit in Mahalas (,Grätzel, Viertel') aufgeteilt. Auch Muktar (,Hauptmann, Vorsteher') war ein gängiger osmanischer Verwaltungsterminus. Durch die Beibehaltung dieser gängigen Termini wollte die österreichisch-ungarische Verwaltung in der Okkupationsphase eine, zumindest oberflächliche, Anknüpfung an die vorhandene lokale Verwaltungstradition herstellen, insbesondere in Sarajevo, wo die Mehrheit der Bevölkerung muslimisch war und in den ersten Okkupationsjahren der österreichisch-ungarischen Verwaltung skeptisch gegenüberstand.

Wesentlich öfter wurden jedoch Lexeme aus der österreichischen Verwaltungssprache ins Bosnische transferiert, u. a. advokat (in der deutschen Version des Textes Advocat), agenda (Agenda), eksekutivni (exekutiv), fond (Fond), inštrukcija (Instruction), konkurs (Concurs), konštituirati (constituieren), magistratski (Magistrats-), štatut (Statut), škontrovati (scontriren) usw. Mit der österreichisch-ungarischen Verwaltung werden viele Internationalismen, insbesondere Latinismen, durch die Vermittlung des österreichischen Deutsch ins Bosnische transferiert, dabei handelt es nicht selten um den so genannten Zivilisationswortschatz, dessen Verwendung Bosnien-Herzegowina von einem osmanisch geprägten an einen mitteleuropäisch geprägten Kulturkreis heranführen sollte (mehr darüber vgl. Memić 2018: 366).

\section{SCHLUSSFOLGERUNG}

Mit der österreichisch-ungarischen Verwaltung ändert sich die Verwaltungsstruktur in Bosnien-Herzegowina grundlegend. Dies macht sich insbesondere durch den Zuzug zahlreicher k. u. k. Beamter und die Einführung einer Verwaltungsstruktur nach dem österreichischen Modell im okkupierten Land bemerkbar. Diese Verwaltungsstruktur 
und ihre deutschsprachige Terminologie ist anhand des analysierten Sprachmaterials aus dem Gemeindestatut für die Stadt Sarajevo (1884) im Grunde österreichisch, wobei man auch gelegentlich Übernahmen aus dem Bosnischen registrieren kann, insbesondere bei landes- und kulturspezifischen Lexemen, um - zumindest in den ersten Okkupationsjahren - eine gewisse Anknüpfung an die vorhandene Verwaltungstradition aus der osmanischen Zeit zu signalisieren.

\section{Primärliteratur}

„Gemeinde-Statut für die Stadt Sarajevo." Sammlung der Gesetze und Verordnungen für Bosnien und die Hercegovina 1 (1884), 1-24.

\section{Sekundärliteratur}

DŽAJA, Srećko (1994) Bosnien-Herzegowina in der österreichisch-ungarischen Epoche (1878-1918). Die Intelligentsia zwischen Tradition und Ideologie. München: R. Oldenbourg.

JUZBAŠIĆ, Dževad (2002) Politika i privreda u Bosni i Hercegovini pod austrougarskom upravom. Sarajevo: Akademija nauka i umjetnosti Bosne i Hercegovine.

MALCOLM, Noel (2011 [1994, 1996]) Bosna: Kratka povijest. Übers. von Zlatko Crnković und Senada Kreso. Sarajevo: Buybook.

MEMIĆ, Nedad (2018) „Diese Worte sind [...] bereits gang und gäbe‘. Zur Internationalisierung des bosnischen Wortschatzes nach der k. u. k. Okkupation." In: C. Ruthner/T. Scheer (Hrsg.), Bosnien-Herzegowina und Österreich-Ungarn, 18781918: Annäherungen an eine Kolonie. Tübingen: Narr Francke Attempto Verlag, 359-372.

NEWEKLOWSKY, Gerhard (2004) „Das Bosnisch-herzegowinische Verfassungsgesetz (Ustavni zakon Vilajeta bosanskog) 1866“. In: R. Katičić (Hrsg.), „Herrschaft “ und „Staat": Untersuchungen zum Zivilisationswortschatz im südosteuropäischen Raum 1840-1870; Eine erste Bilanz. Wien: Verlag der Österreichischen Akademie der Wissenschaften, 29-40.

OKUKA, Miloš (1998) Eine Sprache - viele Erben: Sprachpolitik als Nationalisierungselement in Ex-Jugoslawien. Klagenfurt/Wien: Wieser.

RADANOVIĆ, Sanja (2013) Metode u udžbenicima njemačkog jezika u Bosni i Hercegovini za vrijeme vladavine Austrougarske monarhije. Banja Luka: Filološki fakultet.

ŠATOR, Muhamed (2004) Bosanski/hrvatski/srpski jezik u BiH do 1914. godine. Mostar: fhn.

ŠIPKA, Milan (2001) Standardni jezik i nacionalni odnosi u Bosni i Hercegovini (1850 - 2000): Dokumenti. Sarajevo: Institut za jezik u Sarajevu. 


\section{Zusammenfassung \\ BESONDERHEITEN DER DEUTSCHEN VERWALTUNGSSPRACHE IM K. U. K. BOSNIEN-HERZEGOVINA}

Ziel dieser Arbeit ist, einige wichtige Charakteristika der deutschen Verwaltungssprache in Bosnien-Herzegowina während der österreichisch-ungarischen Zeit (1878-1918) zu identifizieren und einer Analyse zu unterziehen. Nach der Okkupation Bosnien-Herzegowinas führte Österreich-Ungarn grundlegende Reformen des Verwaltungssystems im Okkupationsgebiet durch und engagierte dort fast ausschließlich österreichische und ungarische Staatsbürger als Beamte. Die deutsche Sprache spielte dabei eine wesentliche Rolle als Amts- und Verwaltungssprache, insbesondere im sogenannten inneren Parteienverkehr (Kommunikation innerhalb der Behörden bzw. die Korrespondenz zwischen den bosnisch-herzegowinischen Landesbehörden und dem für die Verwaltung Bosnien-Herzegowinas zuständigen Gemeinsamen Finanzministerium Österreich-Ungarns in Wien). Die deutsche Verwaltungssprache im k. u. k. Bosnien-Herzegowina zeigt eindeutig viele Besonderheiten der österreichischen Amtssprache - besonders im terminologischen Bereich. Es beinhaltet aber auch einige bosnische kulturspezifische Übernahmen bzw. Lehnwörter. Diese sind unter anderem als ein formaler Wunsch zu betrachten, an die eher schwach ausgeprägte Verwaltungstradition aus der osmanischen Zeit in Bosnien-Herzegowina anzuknüpfen.

Schlüsselwörter: Bosnien-Herzegovina, Deutsch, Bosnisch, Sprachkontakt, Verwaltungssprache

\section{Abstract \\ SOME FEATURES OF GERMAN ADMINISTRATIVE LANGUAGE IN THE AUSTRO-HUNGARIAN BOSNIA AND HERZEGOVINA}

This paper aims at discussing some prominent features of the German administrative language in Bosnia and Herzegovina during the Austro-Hungarian period (18781918). After the occupation of Bosnia-Herzegovina, Austria-Hungary conducted comprehensive reforms of the administrative system and employed numerous civil servants from the Austrian and the Hungarian parts of the Monarchy in the occupied country. The German language played an important role as the administrative language, especially in the internal operations of the administration. Administrative German in Bosnia and Herzegovina during the Austro-Hungarian time shows strong features of Austrian administrative terminology, but contains also some Bosnian cultural loanwords which can be interpreted as a formal wish to connect with existing, though rather weak administrative tradition in Bosnia and Herzegovina from the Ottoman period.

Keywords: Bosnia and Herzegovina, German, Bosnian, language contact, administrative language 


\section{Povzetek \\ POSEBNOSTI NEMŠKEGA URADOVALNEGA JEZIKA V C.-KR. BOSNI IN HERCEGOVINI}

Cilj študije je identificirati in analizirati nekatere pomembne značilnosti nemškega uradovalnega jezika v Bosni in Hercegovini v avstro-ogrskem času (1878-1918). Po okupaciji Bosne-Hercegovine je Avstro-Ogrska izpeljala temeljne reforme sistema uprave na okupacijskem območju in tam kot uradnike zaposlila skorajda izključno avstrijske in madžarske državljane. Pri tem je nemščina imela pomembno vlogo kot upravni in uradovalni jezik, še posebej v internem prometu administracije (komunikacija med upravnimi organi oz. korespondenca med bosansko-hercegovskimi deželnimi uradi in skupnim finančnim ministrstvom na Dunaju, pristojnim za upravo Bosne in Hercegovine). Nemški uradovalni jezik v c.-kr. Bosni in Hercegovini nedvomno izkazuje veliko posebnosti avstrijskega uradnega jezika - še posebej na področju terminologije. Vsebuje pa tudi nekatere bosanske kulturnospecifične prevzete besede oz. izposojenke, ki pa jih je mogoče med drugim razumeti kot formalno željo po navezavi na bolj šibko upravno tradicijo v Bosni in Hercegovini iz osmanskih časov.

Ključne besede: Bosna in Hercegovina, nemščina, bosanščina, jezikovni stik, uradovani jezik 